\title{
Role of Serum Prolactin as A Cardiovascular Risk in Type 2 Diabetes Mellitus Patients in Zagazig University Hospitals Lamiaa Abd-Elwahab Mohamed ${ }^{1}$, Essam El Din Mahmoud Lotfy Omer ${ }^{2}$, Sherihan Ahmed Mortada Ibrahim ${ }^{1}$ Departments of ${ }^{1}$ Clinical Pathology and ${ }^{2}$ Internal Medicine, Faculty of Medicine - Zagazig University, Egypt \\ *Corresponding Author: Sherihan Ahmed Mortada Ibrahim, Mobile: (+20) 01064142133, Email: sherihan_mortada@yahoo.com
}

\begin{abstract}
Background: Type 2 diabetes is a global public health crisis that threatens the economies of all nations, particularly developing countries' Hyperprolactinemia may reflect neuroendocrine stress reaction to acute coronary syndromes, which induces acute endothelial dysfunction, insulin resistance, and induction of vascular immune reactions; thus, prolonged hyperprolactinemia lead to arteriosclerosis, augmentation of arterial stiffness, and hypertension.

Objective: To assess role of prolactin as a cardiovascular risk in type 2 diabetes mellitus patients in Zagazig University Hospitals.

Patients and Methods: This study was performed in Clinical Pathology, Cardiology and Internal Medicine Departments Zagazig University Hospitals in the period between July and October 2019. Approval for study was obtained from research administration and research ethics committee, Faculty of Medicine Zagazig University.

Results: Regarding prolactin level, there was a statistically significant increase on comparing group 1 with group 2, 3 and $4(\mathrm{P}=0.016, \mathrm{P}<0.001$, respectively). Also, there was a statistically significant increase on comparing group 2 with group 3 and 4 ( $\mathrm{p}<0.001)$, in addition to significant increase on comparing group 3 with $4(\mathrm{p}=0.012)$. In the present study, serum prolactin increased in patients with acute myocardial infarction (AMI) either in group1 (MI and DM) (mean $=26 \pm \mathrm{SD}=8.09$ ) or group $2(\mathrm{MI})$ (mean $=16.24 \pm \mathrm{SD}=3.37$ ), which points to the possible role of hyperprolactinemia in myocardial infarction. Prolactin was found to be increased as a marker of atherosclerosis so it can be used as biomarker for atherosclerosis or coronary artery disease.

Conclusion: Prolactin can be used as biomarker for coronary artery disease and this is explained by its participation in the process of atherosclerosis. Serum prolactin level increased in acute myocardial infarction.
\end{abstract}

Keywords: Cardiovascular risk, Prolactin, Type 2 diabetes mellitus.

\section{INTRODUCTION}

Type 2 diabetes is a global public health crisis that threatens the economies of all nations, particularly developing countries (1). The American Heart Association has designated diabetes mellitus (DM) as a major risk factor for cardiovascular disease (same category as smoking, hypertension, and hyperlipidemia). Type 2 diabetes patients without a prior myocardial infarction (MI) have a similar risk for coronary artery related events as non-diabetic individuals, who have had a prior $\mathrm{MI}^{(2)}$.

Prolactin is a hormone that is synthesized and secreted from the anterior pituitary lactotrophic cells, which their numbers do not alter with age (3). Hyperprolactinemia may reflect neuroendocrine stress reaction to acute coronary syndromes, which induces acute endothelial dysfunction, insulin resistance, and induction of vascular immune reactions thus; prolonged hyperprolactinemia lead to arteriosclerosis, augmentation of arterial stiffness, and hypertension ${ }^{(4)}$.

Prolactin could also stimulate the atherosclerotic process through its autocrine or paracrine action not through systemic inflammatory induction ${ }^{(5)}$. All these observations showed that a high prolactin level plays a potential role in the development of ischemic cardiac disease, also; hyperprolactinemia leads to dyslipidemia, augmentation of platelets aggregation and amplification of vascular thrombosis that lead to increase in the risk score of acute coronary syndrome ${ }^{(\boldsymbol{(})}$.

The study aimed to assess role of prolactin as a cardiovascular risk in type 2 diabetes mellitus patients in Zagazig University Hospitals.

\section{PATIENTS AND METHODS}

This study was performed in Clinical Pathology, Cardiology and Internal Medicine Departments Zagazig University Hospitals in the period between July and October 2019.

\section{Ethical approval:}

Approval for study was obtained from research administration and Research Ethics Committee, Faculty of Medicine Zagazig University.

Every patient signed an informed written consent for acceptance of the operation. This work has been carried out in accordance with The Code of Ethics of the World Medical Association (Declaration of Helsinki) for studies involving humans.

This study was conducted on 88 male subjects (66 patients and 22 healthy subjects as a control group). 
Subjects in the study were classified into 4 groups:

Group 1: Twenty two diabetic patients with recent myocardial infarction.

Group 2: Twenty two non-diabetic patients with recent myocardial infarction.

Group 3: Twenty two diabetic patients but not known to have acute myocardial infarction.

Group 4: Twenty two healthy subjects (control group). Exclusion Criteria: Patients with renal, hepatic failure, any condition or medication that can cause alteration in the serum prolactin as phenothiazines, butyrophenones and metoclopramide, and type I DM patients.

\section{All subjects enrolled in the study were subjected to the following:}

Clinical assessment include: Detailed history taking and complete clinical examination including pulse, blood pressure temperature, respiratory rate, chest and abdominal examination.

Laboratory investigation include: Determination of serum level of prolactin.

\section{Sample Collection:}

Blood was delivered in plane tube allowed to clot for 30 minutes then centrifuged to separate the serum, which was used for the measurement of prolactin. $1 \mathrm{ml}$ was collected from each subject fasting 8 hours for the measurement of fasting blood glucose.

\section{Limitation and Interference:}

The assay is unaffected by icterus, hemolysis and lipemia. In patients receiving therapy with high biotin no samples should be taken until a minimum of 8 hours after the last biotin does had passed.

Normal level of serum prolactin: Males: 4.6 - 21.4 $\mathrm{ng} / \mathrm{ml}$.

\section{Statistical Analysis}

The collected data were coded, processed and analyzed using the SPSS (Statistical Package for the Social Sciences) version 22 for Windows® (IBM SPSS Inc, Chicago, IL, USA). Data were tested for normal distribution using the Shapiro Wilk test. Qualitative data were represented as frequencies and relative percentages. Chi square test $\left(\chi^{2}\right)$ was used to calculate difference between two or more groups of qualitative variables. Quantitative data were expressed as mean \pm SD (Standard deviation). One way ANOVA was used to compare between means of normally distributed variables (parametric data). $\mathrm{P}$ value $<0.05$ was considered significant.

\section{RESULTS}

This table shows that there was no significant difference between the studied groups as regard age (Table 1).

Table (1): Comparison between the different studied groups according to age

\begin{tabular}{|c|c|c|c|c|c|c|}
\hline Age (years) & $\begin{array}{c}\text { DM with AMI } \\
\text { Group 1 } \\
(\mathbf{n = 2 2})\end{array}$ & $\begin{array}{c}\text { AMI } \\
\text { Group 2 } \\
(\mathbf{n = 2 2})\end{array}$ & $\begin{array}{c}\text { DM } \\
\text { Group 3 } \\
(\mathbf{n = 2 2})\end{array}$ & $\begin{array}{c}\text { Controls } \\
\text { Group 4 } \\
(\mathbf{n = 2 2})\end{array}$ & \multirow{2}{*}{ F } & \multirow{2}{*}{ p } \\
\cline { 1 - 5 } Min. - Max. & $35.0-67.0$ & $34.0-62.0$ & $30.0-70.0$ & $31.0-60.0$ & \multirow{2}{*}{1.412} & \multirow{2}{*}{0.245} \\
\hline Mean \pm SD. & $50.45 \pm 9.13$ & $45.27 \pm 9.89$ & $47.0 \pm 13.18$ & $44.68 \pm 8.05$ & \\
\hline
\end{tabular}

DM: Diabetes mellitus, AMI: acute myocardial infarction, F: ANOVA test. P: Probability

Table (2) and figure (1) show that there was a highly significant difference between the studied groups as regard prolactin level. Prolactin was statistically significantly higher in group 1 when compared with group 2, 3 and 4 . Also, group 2 had a statistically significant increase when compared with group 3 and 4 , in addition to significant increase when compare group 3 with 4 .

Table (2): Comparison between the different studied groups according to prolactin level

\begin{tabular}{|c|c|c|c|c|c|c|c|}
\hline $\begin{array}{c}\text { Prolactin } \\
\text { (ng/ml) }\end{array}$ & $\begin{array}{c}\text { DM with AMI } \\
\text { Group 1 } \\
(\mathrm{n}=\mathbf{2 2})\end{array}$ & $\begin{array}{c}\text { AMI } \\
\text { Group 2 } \\
(\mathbf{n}=\mathbf{2 2})\end{array}$ & $\begin{array}{c}\text { DM } \\
\text { Group } 3 \\
(\mathbf{n}=\mathbf{2 2})\end{array}$ & $\begin{array}{l}\text { Control } \\
\text { Group } 4 \\
(\mathrm{n}=22)\end{array}$ & KW & $\mathbf{p}$ & LSD \\
\hline Mean \pm SD & $26.64 \pm 4.09$ & $\begin{array}{c}16.24 \pm \\
3.37\end{array}$ & $8.85 \pm 1.97$ & $4.97 \pm 1.61$ & 77.486 & $<0.001$ & $\begin{aligned} \mathrm{P} 1 & =0.016 \\
\mathrm{P} 2 & =<0.001 \\
\mathrm{P} 3 & =<0.001 \\
\mathrm{P} 4 & =0.001 \\
\mathrm{P} 5 & =<0.001 \\
\mathrm{P} 6 & =0.012\end{aligned}$ \\
\hline
\end{tabular}

DM: Diabetes mellitus, AMI: Acute myocardial infarction, KW: Kruskal Wallis test

P: Probability, $\mathrm{P}$-value $\leq 0.001$ indicates highly significant

P1: Group 1 vs Group 2

$\mathbf{P}_{\mathbf{2}}$ : Group 1 vs Group 3

$\mathbf{P}_{3}$ : Group 1 vs Group 4

P4: Group 2 vs Group 3

P5: Group 2 vs Group 4

P6: Group 3 vs Group 4

Table (3) and figure (2) show that prolactin level for discrimination of diabetic with AMI and non-diabetics with AMI at cut off value $=20.56 \mathrm{ng} / \mathrm{ml}$. 


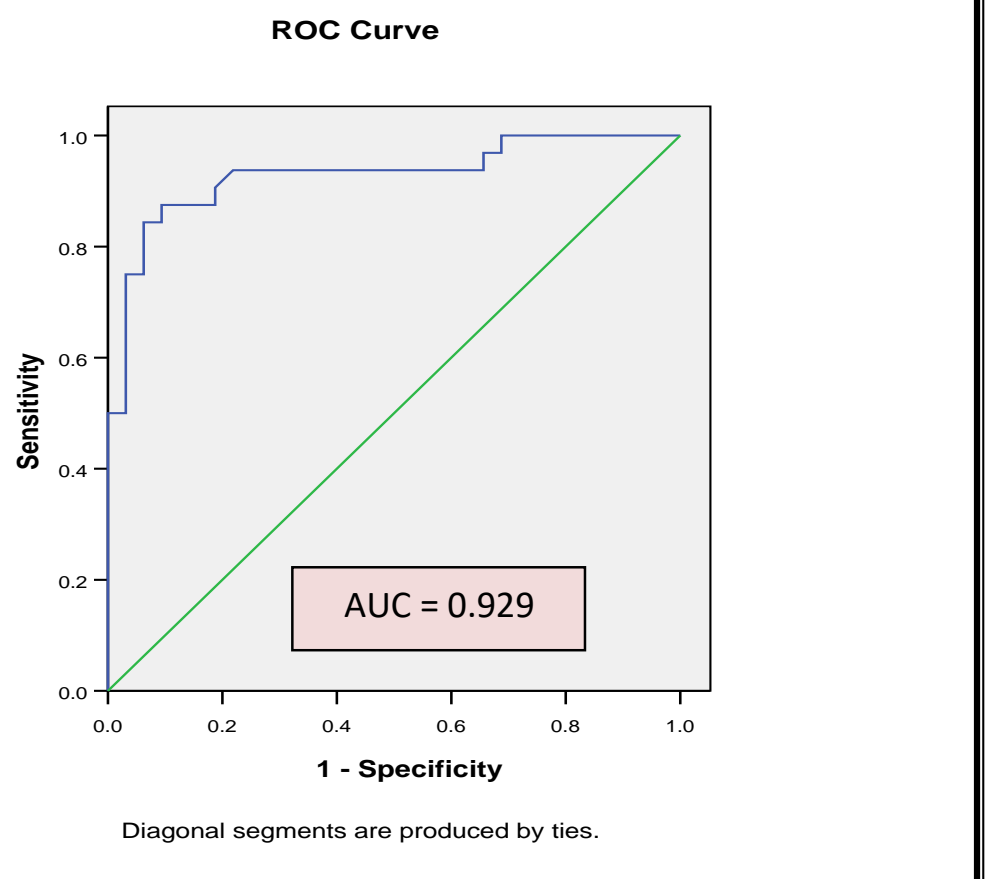

Fig. (2): ROC curve detecting sensitivity and specificity of prolactin level for discrimination of diabetic patients with acute myocardial infarction (AMI) patients and non-diabetics with AMI

Table (3): Prolactin level for discrimination of diabetic patients with acute myocardial infarction (AMI) and non-diabetics patients with AMI

\begin{tabular}{|c|c|c|c|c|c|c|c|}
\hline Variable & Cut off & AUC & Significance & Sensitivity & Specificity & PPV & NPV \\
\hline Prolactin & $\begin{array}{l}20.56 \\
\mathrm{ng} / \mathrm{ml}\end{array}$ & 0.929 & $\mathbf{0 . 0 0 1}$ & $86 \%$ & $92 \%$ & $78.2 \%$ & $95.2 \%$ \\
\hline
\end{tabular}

AUC: area under the curve, PPV: Positive predictive value, NPV: Negative predictive value,

\section{DISCUSSION}

Regarding to age in our study there was no significance difference between the studied groups.

Regarding prolactin level, there was a statistically significant increase in DM with AMI group when compared with AMI, DM and control groups. Also, there was a statistically significant increase in AMI when compared with DM and control, in addition to significant increase in DM when compared with control. In the present study, serum prolactin increased in patients with acute myocardial infarction either in group 1 (MI and DM) or group 2 (MI), which points to the possible role of hyperprolactinemia in myocardial infarction. Whether prolactin is just a biomarker or plays a role in the cardiovascular disease is a matter of discussion.

These findings are in agreement with Wallaschofski et al. ${ }^{(7)}$ who reported that prolactin is a potent cofactor for platelet activation as there's prolactin receptor on human platelets, as increased prolactin value was found to be associated with enhanced P-selectin expression on platelets in patients with acute myocardial infarction. P-selectin mediates rolling of platelets and leukocytes on activated endothelial cells. Wallaschofski et al. (7) demonstrated a positive

correlation between prolactin level and platelet activation as increased plasma prolactin potentiates ADP effects on platelet P-selectin expression and platelets aggregation and consequently increases the incidence of thrombosis.

This is in agreement with Wallaschofski $\boldsymbol{e t}$ al. (8) who demonstrated that prolactin values are increased in stroke and myocardial infarction, and that the involvement of hormonal factors in platelets aggregation should have important clinical implication, and this also is in agreement with Raaz et al. ${ }^{(9)}$ who found that patients with acute myocardial infarction showed significantly higher prolactin values compared to control group, and concluded that this data indicates an association between increased prolactin values and enhanced P-selectin expression on platelet in patients with acute myocardial infarction. These data are supported by the findings of Elgayar et al. ${ }^{(5)}$ who found that AMI patients with diabetes had higher prolactin levels than diabetics and control groups. 
Krysiak et al. ${ }^{(10)}$ measured serum prolactin in 72 diabetic males and compared it to 63 healthy males and found that the diabetic group had significantly higher serum prolactin than the control group.

On the other side, Reuwer et al. ${ }^{(11)}$ observed that serum prolactin did not differ between apparently healthy men and women who developed coronary artery disease (CAD) during follow-up and those who remained free of cardiovascular disease, and people with high prolactin did not have a significantly increased risk of developing CAD. They concluded that elevated serum prolactin dose not predict $\mathrm{CAD}$ in the general population. However, prolactin receptors were found in human coronary artery plaques; this observation may induce a role in prolactin within atherosclerotic plaques. They hypothesize that inflammatory environment within advanced atherosclerotic plaques could stimulate the expression of prolactin receptors, or prolactin could also stimulate the atherosclerotic process through its autocrine or paracrine action not through systemic inflammatory induction. They concluded that hyperprolactinemia may be associated with increased risk of myocardial infarction. Prolactin may have dual effect worsening DM, increasing the atherosclerotic process and hence occurrence of AMI, as one of the serious macrovascular complications in diabetics.

On the other hand Hamidi and DavidgePitts ${ }^{(12)}$ reported that the association between hyperprolactinemia and stroke and myocardial infarction is not well investigated, and it's uncertain if prolactin levels were increased before or after the occurrence of the vascular insult, and whether prolactin is triggering platelets activation in these patients or not, as one of the most likely explanations for increased prolactin in these patients might be stress.

Hyperprolactinemia may be associated with increased risk of atherosclerotic process and hence occurrence of AMI, as one of the serious macrovascular complications in diabetics Hyperprolactinemia detected in diabetics having AMI, supports the recent trend of using dopamine agonists, e.g., bromocriptine in treatment of type 2 DM, especially those who had a prior ischemic event (13).

Also, hyperprolactinemia being associated with increased troponin level in AMI patients may be considered a prognostic factor correlated with the extent of myocardial damage. Thus, coexistences of elevation of both cardiac troponin and serum prolactin in acute MI gives an idea about the underlying predisposing factor of occurrences of MI, and the elevated troponin revealed myocardial necrosis due to the coronary vessel complications that appeared in association with dyslipidemia, which is linked to high prolactin level ${ }^{(\mathbf{1 3})}$.

\section{CONCLUSION}

Prolactin can be used as both biomarker and maker for coronary artery disease and this is explained by its participation in the process of atherosclerosis. Serum prolactin level increased in acute myocardial infarction.

\section{REFERENCES}

1. American Diabetes Association. (2010): Diagnosis and classification of diabetes mellitus. Diabetes Care, 33(1): 62-9.

2. D'Agostino R, Russell M, Huse D et al. (2000): Primary and subsequent coronary risk appraisal: new results from the Framingham study. Am Heart J., 139(2 Pt1):272-81.

3. Kokot I, Pawlik-Sobecka L, Placzkowska S et al. (2013): Prolactin as an immunomodulatory factor in psoriatic arthritis. Postepy Hig Med Dosw., 67:1265-72.

4. Medic-Stojanoska M, Icin T, Pletikosic I et al. (2015): Risk factors for accelerated atherosclerosis in young women with hyperprolactinemia. Med Hypotheses, 84:321-6.

5. Elgayar H, Abu-Shady M, Zaki I et al. (2011): Study of serum prolactin and cardiovascular risk in patients with type diabetes mellitus. Society for Endocrinology, 25: 157-163.

6. Henderson D, Doraiswamy P (2008): Prolactin-related and metabolic adverse effects of atypical antipsychotic agents. J Clin Psychiatry, 1:32-44.

7. Wallaschofski H, Kobsar A, Koksch M et al. (2003): Prolactin receptor signaling during platelet activation. Thrombosis and vascular biology. Horm Metab Res., 35:228-35.

8. Wallaschofski H, Kobsar A, Sokolova O et al. (2004): Co-activation of platelets by prolactin or leptinPathophysiological finding and clinical implications. Horm Metab Res., 36:1-6.

9. Raaz D, Wallaschofski H, Stumpf C et al. (2006): Increased prolactin in acute coronary syndromes as putative Co-activator of ADP- stimulated p-selectin expression. Horm Metab Re., 38:767-72.

10. Krysiak R, Kowalcze K, Szkrobka W et al. (2016): The effect of metformin on prolactin levels in patients with drug-induced hyperprolactinemia. European Journal of Internal Medicine, 30: 94-98.

11. Reuwer A, van Eijk M, Houttuijn-Bloemendaal F et al. (2011): The prolactin receptor is expressed in macrophages within human carotid atherosclerotic plaques: a role for prolactin in atherogenesis? Eur Soc Endocrinol., 208:107-17.

12. Hamidi O, Davidge-Pitts C (2019): Transfeminine hormone therapy. Endocrinology and, Metabolism Clinics, 2: 341-355.

13. Islam S, Pathan F, Ahmed T (2015): Clinical and biochemical characteristics of polycystic ovarian syndrome among women in Bangladesh. Mymensingh Med J., 24:310-8. 